\title{
Zur Farbreaktion von Amiloridhydrochlorid Ph. Eur. ${ }^{+}$
}

\author{
Klaus Görlitzer ${ }^{1 *}$, Silke Huth ${ }^{1}$, Peter G. Jones ${ }^{2}$, \\ Edith Gößnitzer ${ }^{3}$ und Winfried Wendelin ${ }^{3}$ \\ ${ }^{1}$ Institut für Pharmazeutische Chemie der Technischen Universität Braunschweig, \\ Beethovenstraße 55, D-38106 Braunschweig, \\ ${ }^{2}$ Institut für Anorganische und Analytische Chemie der Technischen Universität Braunschweig, \\ Hagenring 30, D-38106 Braunschweig, \\ ${ }^{3}$ Institut für Pharmazeutische Chemie und Pharmazeutische Technologie der Karl-Franzens- \\ Universität Graz, Schubertstraße 1, A-8010 Graz
}

The Colour Reaction of Amiloride Hydrochloride Ph. Eur.

The reaction of amiloride hydrochloride $(1 . \mathrm{HCl})$ with bromine in alkaline solution generated a yellow-brown dehydrogenation product, which turned out as 3-(3-amino-1,2,4-oxadiazol5-yl)-5-chloro-2,6-pyrazinediamine (2). The structure was deduced from the MS and the NMR spectra of 2 with the help of comparisons with corresponding spectra of amiloride (1) and reference substances 3 - 5. The agreement of all relevant data of the product and of authentical oxadiazolylpyrazine $\mathbf{2}$ as well as the accomplished X-ray analysis confirmed the postulated structure. The mechanism of the formation of $\mathbf{2}$ is also discussed.

(Keywords: Amiloride, colour reaction with bromine; 2,6-Pyrazinediamine, 3-(3-amino-1,2,4-oxadiazol-5-yl), 5-chloro; X-ray analysis; MS analysis; and NMR analysis)

\section{Einleitung}

Die Pharmacopoea Europaea ${ }^{1}$ lässt zur Prüfung des diuretisch wirksamen Amiloridhydrochlorids (1.HCl, Darstellung und spektroskopische Daten siehe ${ }^{2-4}$ ) auf Identität neben physikalisch-chemischen Methoden auch eine Farbreaktion durchführen. Dazu wird 1.HCl mit Cetrimid (Cetyltrimethylammoniumchlorid)-Lösung, Natronlauge und Bromwasser bei Raumtemperatur umgesetzt. Die grünlichgelbe Färbung der erhaltenen Lösung schlägt auf Zusatz von Salzsäure nach Dunkelgelb um. Dies ist mit einer starken blauen Fluoreszenz verbunden, die durch ultraviolettes Licht der Wellenlänge von $365 \mathrm{~nm}$ angeregt wird.

\footnotetext{
${ }^{+}$Herrn Univ.-Prof. Dr. Dr. h.c. W. Fleischhacker mit den besten Wünschen zum 70. Geburtstag gewidmet.
} 
Die Struktur des für die Farbreaktion verantwortlichen Produkts war bislang unbekannt. Weder in der Monographie in Florey ${ }^{3}$ noch im Kommentar zur Pharmacopoea Europaea ${ }^{5}$ finden sich Hinweise auf die chemischen Vorgänge bei dieser Identitätsprüfung.

\section{Ergebnisse und Diskussion}

Verfährt man nach der Vorschrift der Pharmacopoea Europaea', verzichtet jedoch auf den für die Farbreaktion nicht notwendigen Zusatz von Cetrimid und extrahiert anschließend bei pH 3.2 mit tert-Butylmethylether, so erhält man nach Abdestillieren des Lösungsmittels und Waschen des Rückstandes mit Chloroform einen braunen Feststoff, der beim Umkristallisieren aus Dimethylformamid/Wasser gelbbraune Kristalle liefert. Die Ausbeute beträgt allerdings nur $1 \%$.

Ableitung der Struktur des Farbprodukts 2 durch Vergleich des Massenspektrums mit jenem von Amilorid (1)

Im Massenspektrum wird der Molekülpeak des Farbprodukts bei einer um $\mathrm{m} / \mathrm{z}=2$ geringeren Masse gegenüber dem Edukt Amilorid (1) gefunden. Die Substanz enthält aufgrund des Isotopenmusters weiterhin ein Chloratom. Aus dem Massenspektrum und der Elementaranalyse ergibt sich die Summenformel $\mathrm{C}_{6} \mathrm{H}_{6} \mathrm{ClN}_{7} \mathrm{O}$, die Verbindung ist demnach ein Dehydrierungsprodukt von Amilorid.

Wichtige Hinweise auf die Struktur des Farbprodukts ergeben sich aus dem Vergleich des MS mit jenem von Amilorid (1) (Schema 1). Im MS von 1 erscheint der Molekülpeak $(\mathrm{m} / \mathrm{z}$ 229/231) als Base Peak. Außerdem findet man charakteristische Peaks mit der Masse $\mathrm{M}^{+} \cdot-17, \mathrm{M}^{+} \cdot-42, \mathrm{M}^{+} \cdot-58$ (Pyrazincarbonylium, $m / z$ 171/173, R.I. 34), $\mathrm{M}^{+}-59(\mathrm{~m} / \mathrm{z} 171 / 173$, nicht im Schema), $\mathrm{M}^{+} \cdot-85$ (Pyrazin-Kationen, $m / z$ 144/146, R.I. 50), und $\mathrm{M}^{+} \cdot-86$ (PyrazinylKationen, $m / z$ 143/145, R.I. 20). Die genannten Peaks entstehen durch Eliminierung von Ammoniak bzw. Cyanamid, Diaminomethylenaminyl-, Guanidin, Carbonylguanidin bzw. Amidinoisocyanat, sowie Guanidincarbonyl-Radikale, und dokumentieren den schrittweisen Abbau der Guanidino- bzw. (Diaminomethylenamino)carbonyl-Seitenkette. Komplementär findet man auch intensitätsstarke Peaks für Cyanamid-, Guanidin- und GuanidincarbonylKationen bei $m / z 43$ (nicht im Schema), 59 und 86.

Aus dem Molekülion des Farbstoffs 2 ( $m / z$ 227/229, Base Peak) hingegen entstehen nur zwei wichtige Fragmentionen, nämlich Pyrazincarbonyl- $\left(\mathrm{M}^{+} \cdot-56, m / z\right.$ 171/173, R.I. 56) und Pyrazinyl-Kationen, $\left(\mathrm{M}^{+} \cdot-84, m / z \quad 143 / 145\right.$, R.I. 50), die auch im MS von Amilorid (1) gefunden werden. Sie bilden sich beim Farbstoff 2 durch Abspaltung des vergleichsweise 
wasserstoffarmen Radikals $\mathrm{H}_{2} \mathrm{NCN}_{2} \cdot$ (oder $\mathrm{HCN}, \mathrm{N}_{2}$ und $\mathrm{H}$ ) bzw. von AminooxadiazolylRadikalen. Die Diaminochlorpyrazinteilstruktur bleibt bei der Fragmentierung sowohl des Farbstoffs als auch von 1 weitgehend unberührt (Peaks mit jeweils nur geringer Intensität durch Abspaltung von $\mathrm{HCN}$ aus den Molekülionen sowie den Fragmentionen $\mathrm{M}^{+}$- 56 und $\mathrm{M}^{+} \cdot$-84).

Die genannten Befunde zeigen, daß das Farbprodukt aus Amilorid (1) durch Dehydrierung der Guanidincarbonyl-Teilstruktur ohne Beteiligung des Diaminochlorpyrazinteiles entstanden ist. Da eine Dehydrierung des Guanidincarbonyl-Restes nur unter gleichzeitigem Ringschluß möglich ist, folgt, daß das Produkt sehr wahrscheinlich als 3-(3-Amino-1,2,4oxadiazol-5-yl)-5-chlor-2,6-pyrazindiamin (2) vorliegt (Schema 1). Das Vorhandensein des kompakten aromatischen Aminoxadiazolylrestes in $\mathbf{2}$ an Stelle des leicht fragmentierbaren Guanidincarbonylrestes in 1 erklärt auch sehr gut die im Vergleich zu 1 höhere Stabilität des Farbprodukts 2 im MS.

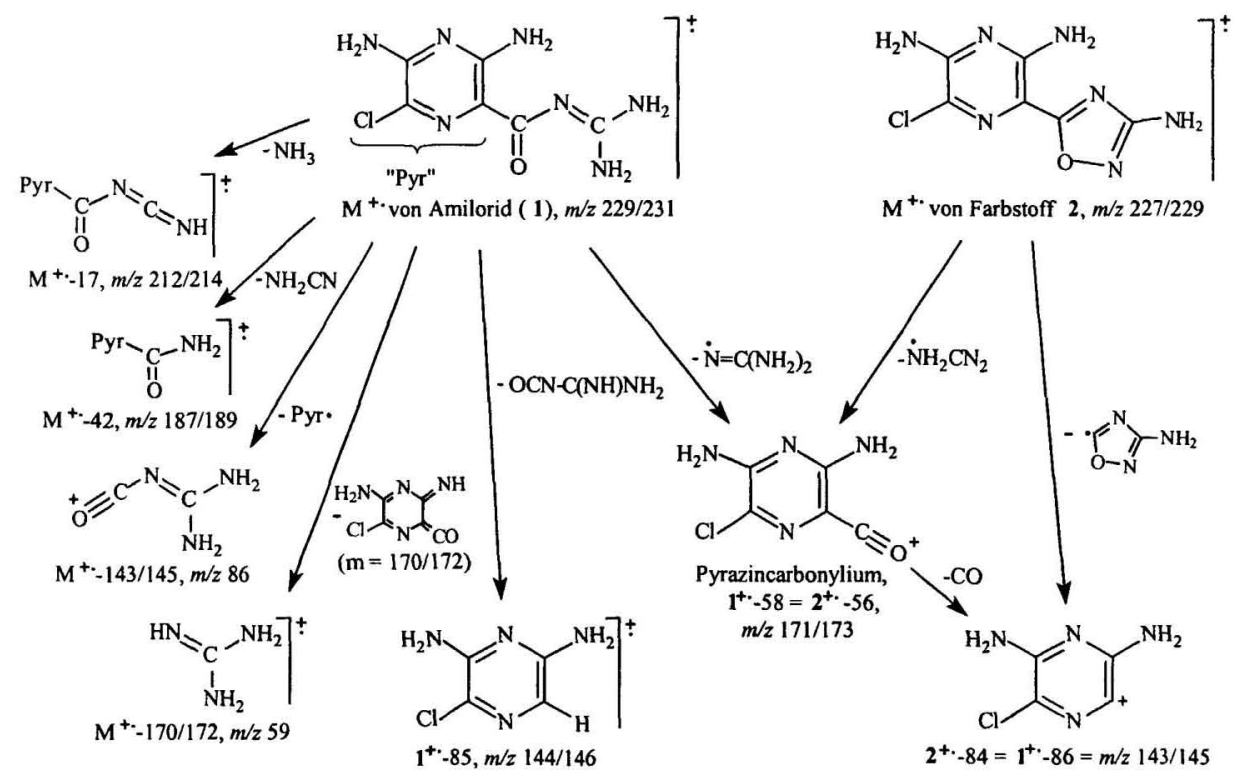

Schema 1. Fragmentierungsreaktionen von Amilorid (1) und Farbstoff 2 
Ableitung der Struktur des Farbstoffes 2 aus den ${ }^{1} H$ - und ${ }^{13} C-N M R$-Spektren anhand von Vergleichen mit Referenzsubstanzen

Das FT-IR-Spektrum zeigt fün separierte NH-Valenzschwingungen, das ${ }^{1} \mathrm{H}-\mathrm{NMR}$ in $\left[D_{6}\right]$ DMSO hingegen nur zwei Signale für Aminprotonen mit den Intensitäten 4 und 2 bei $\delta=7.28$ und 6.26. Die chemische Verschiebung der Protonen zweier chemisch äquivalenter Aminogruppen von $\delta=7.28$ ist typisch für 3/5-Aminopyrazin-2-carbonsäure-Derivate wie z. B. 3-Amino-2-pyrazincarbonitril (3) $(\delta=7.30)^{6}$ oder 3,5-Diamino-5-chlorpyrazin-2-( $N$ cyanocarboxamidin) (4) $(\delta=7.47)^{7}$, was auf das Vorhandensein einer kaum veränderten Pyrazindiamin-Teilstruktur im Reaktionsprodukt hindeutet. Die Protonen der dritten Aminogruppe treten bei $\delta=6.26$ in Resonanz, einem Bereich, der bei Oxadiazolaminen, z. B. 5-Phenyl-1,2,4-oxadiazol-3-amin (6) $(\delta=6.40)^{8}$, beobachtet wird (Schema 2). Der Vergleich der ${ }^{13} \mathrm{C}$-Spektren von Amilorid 1 und Produkt 2 bestätigt diese Befunde, die Spektren unterscheiden sich lediglich bei den chemischen Verschiebungen der C-Atome in der Guanidincarbonyl- bzw. Oxadiazol-Teilstruktur sowie beim Carbonyl-substituierten C-2 von 1 bzw. dem korrespondierenden Oxadiazolyl-substituierten C-3 des Farbstoffs deutlich.

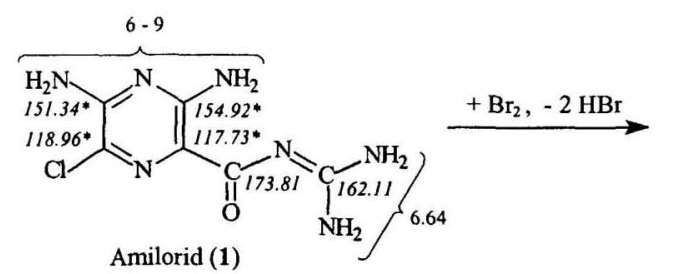<smiles>N#Cc1nccnc1N</smiles>

3<smiles>N#C/N=C(\N)c1nc(N)c(Cl)nc1N</smiles>

4

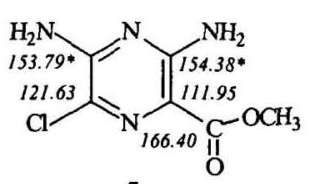

5

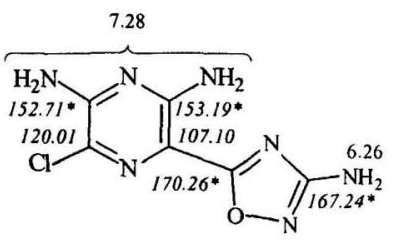

Farbstoff 2

Schema 2. ${ }^{1} \mathrm{H}$ - und ${ }^{13} \mathrm{C}$ (kursiv)-Verschiebungen ( $\delta$-Werte in ppm) von $1^{3,4}, 2$ und Vergleichssubstanzen $3-6^{6-9}$ (alle in [ $\left.\mathrm{D}_{6}\right] \mathrm{DMSO}$, die Zuordnung bei mit * markierten Werten ist nicht gesichert)

Die chemischen Verschiebungen der Pyrazinkohlenstoffe C-2,6 ( $\delta=153.19 / 152.71)$ und C-5 $(\delta=120.01)$ von Farbstoff 2 stimmen weitgehend mit denen der Signale für die korrespondieren C-3,5 und C-6 bei $1(\delta=154.92 / 151.34 ; 118.96)$ bzw. Aminopyrazincarbonsäureester $5(\delta=154.38 / 153.79 ; 121.63)^{9}$ überein (siehe Schema 2$)$. 
Diese Befunde zeigen, ebenso wie die MS-Vergleiche, daß die Reaktion von 1 mit Brom unter Dehydrierung lediglich des Guanidincarbonylrestes erfolgt war und der Farbstoff als (Aminooxadiazolyl)chlorpyrazindiamin 2 vorliegt.

Zur Sicherung der Struktur von 2 wurden eine Röntgenstrukturanalyse und 2D-NMRUntersuchungen durchgeführt. Parallel dazu wurde in der Literatur geprüft, ob Verbindung 2 bereits bekannt ist.

\section{Röntgenstrukturanalyse von 2}

Zur Durchführung wurden Einkristalle gezüchtet und eine Röntgenkristallstrukturanalyse durchgeführt, die bewies, dass eine Dehydrierung des Acylguanidins von 1 zum 3-Amino1,2,4-oxadiazol 2 erfolgt war (Abb. 1). Wie die Torsionswinkel zeigen, liegen der Pyrazinund der Oxadiazol-Ring praktisch in einer Ebene. Die nahezu planare Fixierung durch Wasserstoffbrückenbindung (der Abstand $\mathrm{N}(3)-\mathrm{H} \cdots \mathrm{N}(6)$ beträgt $2.18 \AA$ ) zwischen der 2-Aminogruppe des Pyrazins und dem N-4 des 1,2,4-Oxadiazols dürfte für die intensive blaue Fluoreszenz des Farbprodukts in Lösung verantwortlich sein.

\section{Literaturrecherche}

Farbstoff 2 ist bekannt und bereits auf zwei alternativen, jeweils eindeutigen Wegen ${ }^{7,10}$ synthetisiert worden, z. B. von Watthey et al. ${ }^{7}$ durch Addition von Hydroxylamin an das Pyrazin-2-(N-cyancarboxamidin) 4 (Schema 2) und Ringschluß zu 2. Der Vergleich der ${ }^{1} \mathrm{H}$ - und ${ }^{13} \mathrm{C}$-Verschiebungen im NMR sowie der IR-Banden des von uns isolierten Farbstoffs mit den Daten des von Bock et al. ${ }^{10}$ hergestellten Oxadiazolylpyrazindiamins 2 ergab eine gute Übereinstimmung. Verbindung 2 ist als Prodrug von Amilorid (1) anzusehen, da aus der Substanz 2 in Tiermodellen in vivo durch reduktive Ringöffnung 1 gebildet wird ${ }^{10}$. Synthesen von Oxadiazolaminen des Typs 2 durch Dehydrierung von Acylguanidinen, wie hier bei der Reaktion von 1 mit Brom und Natronlauge beobachtet, sind ebenfalls beschrieben worden. Z. B. läßt sich das als Vergleichssubstanz erwähnte 5-Phenyl-1,2,4-oxadiazol2-amin (6) durch Behandlung von Benzoylguanidin mit Natriumhypochlorit herstellen ${ }^{11}$.

\section{D-NMR-Untersuchungen von 2}

Wie oben gezeigt, erlaubten die Vergleiche der ${ }^{1} \mathrm{H}$ und ${ }^{13} \mathrm{C}-\mathrm{NMR}$-Spektren des isolierten Produkts 2 mit den Spektren von Amilorid (1) und den Referenzsubstanzen 3 - 6 die Ableitung der Struktur des Aminooxadiazolylpyrazindiamins 2. Offen blieb allerdings die Frage 


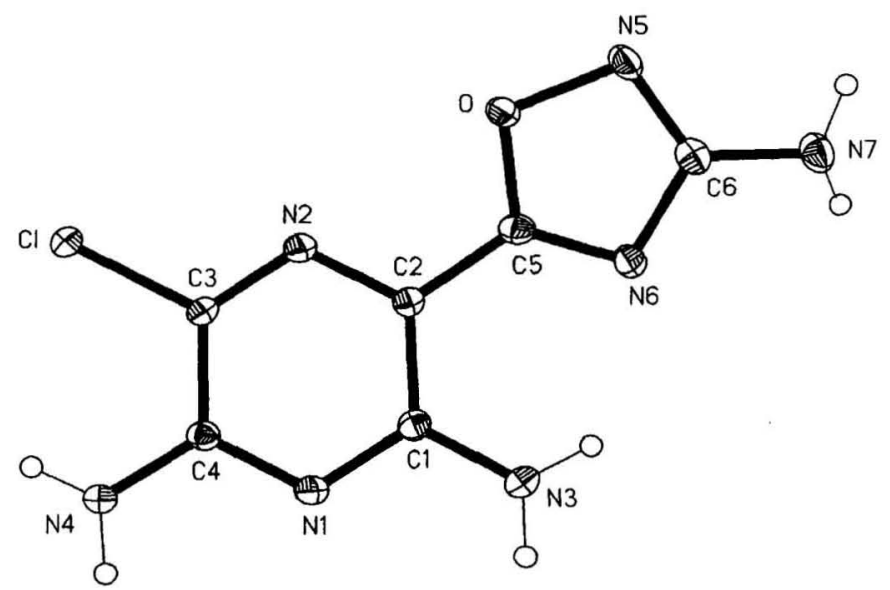

Kristalldaten und Strukturverfeinerung

Summenformel

$M$ r

Temperatur

Wellenlänge

Kristallsystem

Raumgruppe

Gitterkonstanten

Volumen, Z

Dichte (berechnet)

Absorptionskoeffizient

$\mathrm{F}(000)$

Kristallgröße

$\theta$-Bereich für die Datensammlung

Limitierende Indizes

Zahl der Reflexe

Unabhängige Reflexe

Vollständigkeit bis $\theta=30^{\circ}$

Verfeinerungsmethode

Daten/Einschränkungen/Parameter

Goodness-of-fit on $\mathrm{F}^{2}$

Final R-Indizes [l>2 $\sigma(\mathrm{I})]$

R-Indizes (alle Daten)

Größte Differenzpeaks

(Maximum und Minimum)

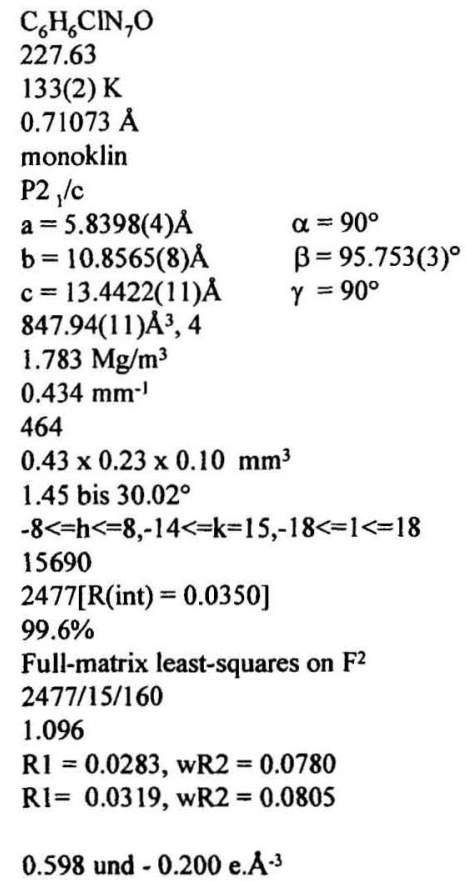

0.598 und $-0.200 \mathrm{e} . \AA^{-3}$

Abb.1 Röntgenstrukturanalyse des 3-(3-Amino-1,2,4-oxadiazol-5-yl)-5-chlor-2,6-pyrazindiamins (2). Ellipsoide entsprechen 50\%iger Aufenthaltswahrscheinlichkeit. 
der korrekten Zuordnung der jeweils paarweise nahe beieinander liegenden ${ }^{13} \mathrm{C}-\mathrm{NMR}$-Signale bei $\delta=153.19 / 152.71 \mathrm{zu} \mathrm{C}-2$ bzw. C-6 und bei $\delta=107.10 / 120.01 \mathrm{zu} \mathrm{C-3} \mathrm{bzw.} \mathrm{C-5} \mathrm{im}$ Pyrazin-, sowie der Signale bei $\delta=167.24 / 170.26$ zu C-3' bzw. C-5' im Oxadiazolring. Vergleiche mit Amilorid (1), dessen ${ }^{13} \mathrm{C}$-Signale Smith et al. ${ }^{4}$ auf Grund sorgfältiger Studien mit modifizierten Modellsubstanzen des Typs $1(\mathrm{H}, \mathrm{Br}$, J anstelle von $\mathrm{Cl}$; Dimethylamino anstelle von $\mathrm{NH}_{2}$, etc.), jedoch ohne Absicherung durch Korrelationsspektren zugeordnet hatten, und mit 6-Chlor-3,5-diaminopyrazin-2-carbonsäuremethylester (5) ${ }^{9}$ (siehe Schema 2) sprachen für die Zuordnung der Signale von 2 bei $\delta=107.10$ und 120.01 zu C-3 und C-5. Sie erlaubten jedoch nicht die sichere Zuordnung der beiden anderen Signalpaare.

Es wurde deshalb versucht, die Zuordnung der ${ }^{13} \mathrm{C}$-Signale von 2 über die Messung von $\mathrm{CH}-$ Korrelationen zwischen den zwei Sorten von Aminprotonen in 2,6-Stellung des Pyrazinringes ( $\delta=7.28, \mathrm{I}=4) \mathrm{bzw}$. in 3-Stellung des Oxadiazolringes $(\delta=6.26, \mathrm{I}=2)$ und Kohlenstoffen der beiden Ringe über zwei, drei und vier Bindungen durchzuführen. Zugleich sollte durch Auffinden überlappender Korrelationen im Bereich von C-3 und C-5' die Verknüpfung von Pyrazin- und Oxadiazolring spektroskopisch nachgewiesen werden.

Die zunächst in $\left[\mathrm{D}_{6}\right] \mathrm{DMSO}$ durchgeführten $\mathrm{HMBC}$ (heteronuclear multiple bond correlation) Experimente zeigten jedoch nur Korrelationen zwischen den Aminprotonen bei $\delta=7.28$ und den Kohlenstoffatomen des Pyrazininges bei $\delta=107.10$ und 152.71, was für die beabsichtigte detaillierte Strukturanalyse nicht genügte.

Um eine Nichtäquivalenz der Aminogruppen am Pyrazin herbeizuführen und zusätzliche $\mathrm{CH}-$ Korrelationen $\mathrm{zu}$ finden, wurden auch Messungen in Lösungsmittelgemischen (ein kleiner Anteil an $\left[\mathrm{D}_{6}\right] \mathrm{DMSO}$ war wegen der sehr schlechten Löslichkeit von 2 in lipophilen Lösungsmitteln immer notwendig) und in Gegenwart von Säuren durchgeführt.

Die Verwendung von $\left[\mathrm{D}_{6}\right]$ DMSO-[ $\left.\mathrm{D}_{6}\right]$ Benzol $(1: 6)$ als Lösungsmittel führte bei den Signalen für die Aminprotonen des Pyrazin-2,6-diamins bzw. 3'-Aminooxadiazolylrestes von 2 zu Tieffeldverschiebungen von 0.54 und 0.26 bzw. $0.35 \mathrm{ppm}$, sodaß nun für die Aminogruppen des Pyrazindiamins zwei getrennte Resonanzen bei $\delta=7.82$ und 7.54 auftraten (Schema 3). Allerdings waren die beiden Signale durch chemischen Austausch stark verbreitert, sodaß keine erfolgreichen 2D-Messungen möglich waren. Die Anwendung von $\left[\mathrm{D}_{6}\right] \mathrm{DMSO}-\mathrm{CDCl}_{3}$ (1:6) hingegen führte bei den genannten Aminprotonen zu Hochfeldverschiebungen der Signale von -0.17 und -0.37 bzw. -0.35 ppm bei nur mäßiger Verbreiterung. Wechselseitige NOEs zwischen den Oxadiazolamin-Protonen bei $\delta=5.91$ und den Aminprotonen bei $\delta=7.11$ ermöglichten die Zuordnung des letztgenannten Signals zu den $\mathrm{NH}_{2}$ Protonen in Position 2 des Pyrazindiamins (Schema 3). CH-Korrelationen waren aber auch in diesem Falle nicht zu beobachten. 


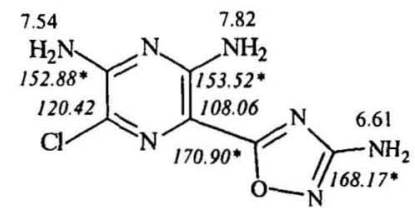

2, Werte in $\left[\mathrm{D}_{6}\right]$ DMSO / [ $\left.\mathrm{D}_{6}\right]$ Benzol (1:6)

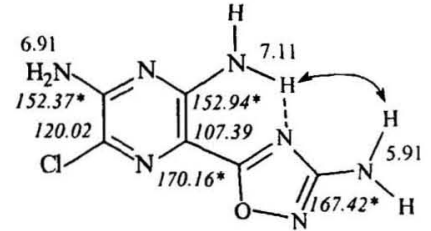

2, Werte in $\left[\mathrm{D}_{6}\right] \mathrm{DMSO} / \mathrm{CDCl}_{3}(1: 6)$

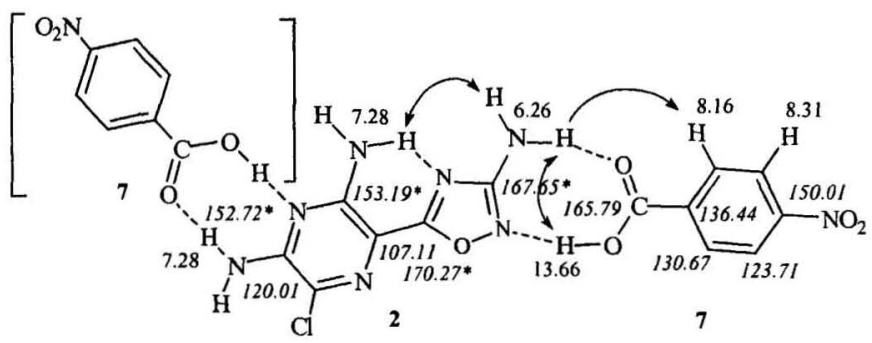

Assoziat aus 2 und 7 (1:1) und [1:2], Werte des (1:1)-Komplexes in [D]DMSO

Schema 3. ${ }^{1} \mathrm{H}$ - und ${ }^{13} \mathrm{C}($ kursiv)-Verschiebungen ( $\delta$-Werte in ppm) sowie NOEs (symbolisiert durch Pfeile) von $\mathbf{2}$ in verschiedenen Lösungsmitteln und von Assoziaten aus $\mathbf{2}$ und $\mathbf{7}$ in

$\left[D_{6}\right]$ DMSO. Die Zuordnung von mit * bezeichneten Werten ist nicht gesichert.

In drei weiteren Experimenten wurde geprüft, welche Auswirkungen auf Lage und Form der $\mathrm{NH}_{2}$-Signale die Zugabe von Säuren hat, die Wasserstoffbrücken bilden.

Die ${ }^{1} \mathrm{H}$ - und ${ }^{13} \mathrm{C}-\mathrm{NMR}$ wie auch die $\mathrm{CH}-$ Korrelationsspektren der (1:1)- und (1:2)Mischung von 2 und $p$-Nitrobenzoesäure (7) in $\left[\mathrm{D}_{6}\right] \mathrm{DMSO}$ waren fast identisch mit den durch Superposition der Einzelspektren beider Verbindungen herstellbaren; 2 bildete also mit 7 kein Salz. Wechselseitige NOEs zwischen den Oxadiazolamin-Protonen bei $\delta=6.26$ und den sauren Protonen von 7 bei $\delta=13.66$ deuten im (1:1)-Gemisch auf die Bildung von Assoziaten aus 2 und 7 hin, die, wie in Schema 3 skizziert, über Wasserstoffbrücken verbunden sein dürften. Übereinstimmend damit führt die Einstrahlung auf das Signal bei $\delta=6.26$ zur Anregung der $o$-Protonen von 7 bei $\delta=8.16$, und zudem, wie bei Base 2, der Aminprotonen in 2-Stellung des Pyrazins bei $\delta=7.28$. Ebenfalls wie bei 2 findet man $\mathrm{CH}$ Korrelationen zwischen den Pyrazinamin-Protonen und C-3 $(\delta=107.11)$ sowie C-2 bzw. C-6 $(\delta=152.72)$. Eine schwache Korrelation zwischen den Oxadiazolaminprotonen bei $\delta=6.26$ und dem C-Signal bei $\delta=170.27$ zeigt, daß dieses Signal C-3' oder C-5' im Oxadiazolring zuzuordnen ist. 
Für die (erwartete) Bildung eines (1:2)-Komplexes mit zusätzlichen Wasserstoffbrücken zwischen einer der beiden Pyrazinamin-Teilstrukturen von $\mathbf{2}$ und der Carboxylgruppe eines zweiten Moleküls von 7 (siehe Schema 3) wurden keine Hinweise gefunden.

Bei äquimolarer Zugabe von Trifluoressigsäure zur (1:1)-Mischung von 2 und 7 in $\left[D_{6}\right]$ DMSO bildete sich ebenfalls kein Salz. Dies zeigte die unveränderte Lage der Signale für 2 und $7 \mathrm{im}{ }^{13} \mathrm{C}$-Spektrum. Im ${ }^{1} \mathrm{H}$-Spektrum bleibt die chemische Verschiebung der 0 und $m$-Protonen von 7 ebenfalls unverändert ( $\delta=8.15$ und 8.30), doch erscheinen hier für die Aminprotonen nun sehr breite Signale im Bereich von $\delta=6.8-8.0(\mathrm{I}=2)$ und $\delta=9.8$ $12.8(I=5-6)$.

\section{Diskussion des Reaktionsverlaufes}

Wie erwähnt, reagiert Amilorid (1) mit Brom und Natronlauge unter Dehydrierung des Guanidincarbonylrestes zum Oxadiazolylpyrazin 2. Sehr wahrscheinlich entsteht dabei intermediär das 1-Brom-2-(pyrazincarbonyl)guanidin 8I. Die basenkatalysierte Abspaltung eines Protons induziert dann die Cyclisierung zum Oxadiazolamin 2 (Schema 4). Die genauere Analyse der möglichen Reaktionsabläufe zeigt, daß die Weiterreaktion von 8I bzw. des tautomeren 1-Brom-3-acylguanidins 8II ohne Beteiligung des Diaminopyrazinylrestes alternativ auch durch Umlagerung eines Restes, ähnlich wie beim Hofmann-Abbau, eingeleitet werden könnte. Die baseninduzierte Wanderung des Pyrazinylrestes von 8I bzw. des Acylamidorestes von 8II zum bromsubstituierten N-1 sowie Abspaltung von Bromid führt ausgehend von 8I zum 1-Carbonyl-2-pyrazinylguanidin 9 (einem Amidinoisocyanat) bzw. von 81 zum Amidocarbodiimid 11. Aus 11 könnte sich durch basenkatalysierten Ringschluß, ohne Beteiligung des Diaminopyrazinylrestes, das 1,3,4-Oxadiazol-2-amin 12, ein Stellungsisomeres des 1,2,4-Oxadiazol-3-amins 2 bilden. 12 kann auch direkt aus der Bromverbindung 8II, durch Aneinanderreihung der Umlagerung und des Ringschlusses, in einem Zug entstehen.

Die stellungsisomeren Pyrazinotriazepinone 13 bzw. 10 und 14 (IUPAC-Namen in Schema 4), könnten sich durch Cyclisierung der Intermediate 8II bzw. 9 und 11 bilden. Anlagerung der Aminogruppen in 3-Stellung der Pyrazine an N-2 (oder an den bromsubstituierten Stickstoff $\mathrm{N}-1$ ) des Guanidinorestes von 8 II (und Eliminierung von Bromid) gibt 13, Addition der Pyrazinaminogruppe an den Isocyanat-Kohlenstoff von 9 führt zu Produkt 10, und bei analoger Cyclisierung von 11 unter Beteiligung des CarbodiimidKohlenstoffs entsteht 14. Da aber bei diesen Ringschlüssen die Diaminopyrazin-Teilstruktur 


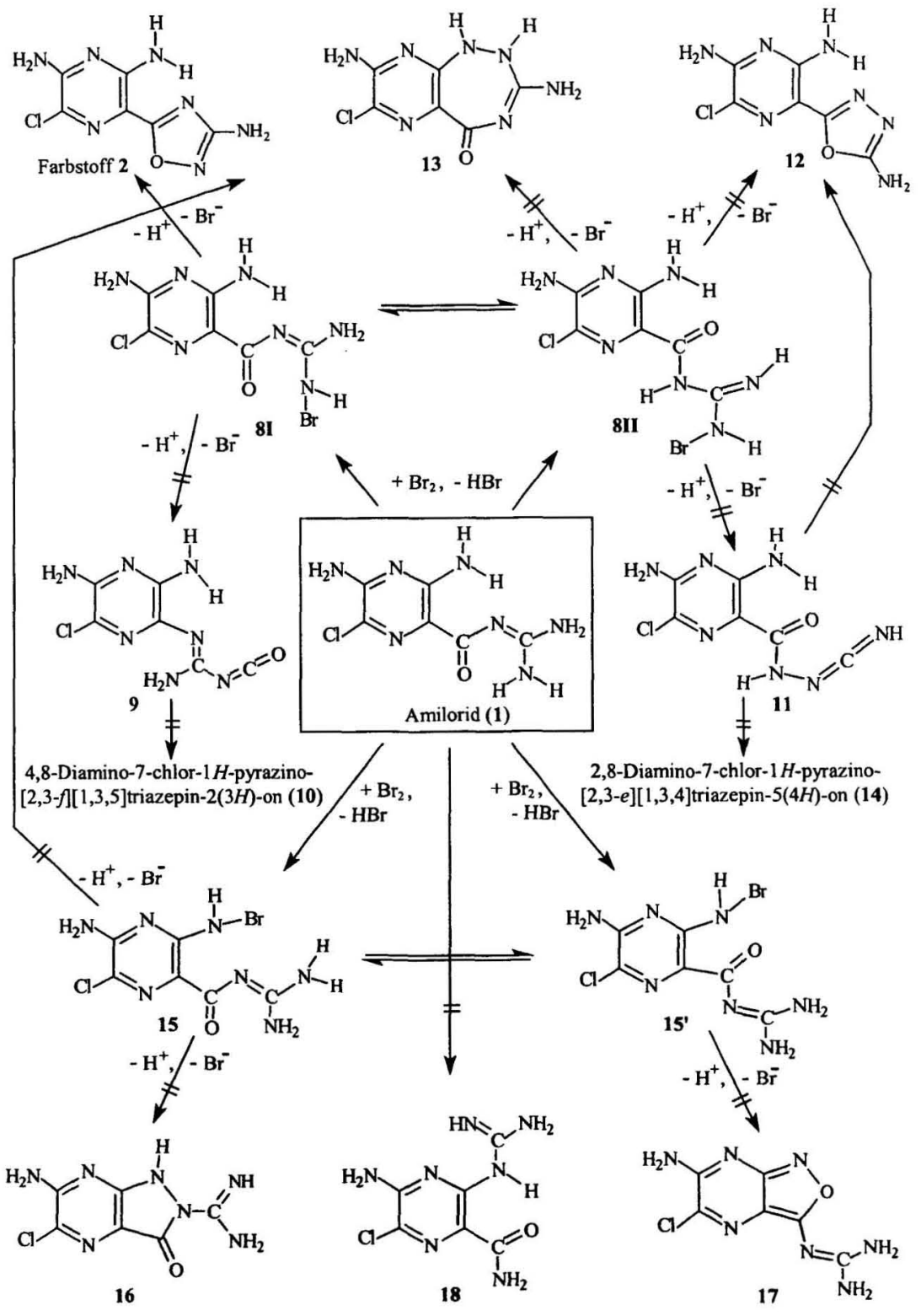

Schema 4. A priori mögliche Reaktionen bei der Umsetzung von Amilorid (1) $\mathrm{mit}^{\mathrm{Br}} \mathrm{Br}_{2} / \mathrm{NaOH}$ 
verlorengeht, waren 13, 10 und 14 als mögliche Strukturen für das Farbprodukt auszuscheiden. Auch die Bildung eines reaktiven 3-Bromaminopyrazins 15 als Intermediat war vorstellbar. Ausgehend von den Isomeren 15 bzw. 15' hätten sich durch Bromwasserstoffabspaltung und Ringschluß Verbindungen wie das Pyrazolopyrazincarboxamidin 16 bzw. das Isoxazolopyrazinylguanidin 17, oder aus $15 \mathrm{I}$ auch das schon erwähnte Pyrazinotriazepinon 13 bilden können.

Außerdem kam für die Einleitung der Reaktion auch die Übertragung eines Amidinorestes vom Guanidin auf die 3-Aminogruppe des Amilorid (1) in Frage. Die Cyclodehydrierung des gebildeten 3-Guanidino-2-pyrazincarboxamids 18 konnte dann zu einem Isoxazolooder Pyrazolopyrazincarboxamidin führen, der Hofmann-Abbau des Amids 18 hingegen zu einem Isocyanat und dessen Ringschluß zu einem Imidazopyrazincarboxamidin (Folgeprodukte nicht im Schema 4). Schließlich war auch die Öffnung des Pyrazinringes von 1 unter Bildung eines Triaminopyrimidinons (Produkt nicht im Schema 4) denkbar.

Das tatsächliche Eintreten der beiden letztgenannten Reaktionsfolgen schien allerdings in Anbetracht der angewendeten sehr milden Reaktionsbedingungen wenig wahrscheinlich. Da auch bei diesen Reaktionen die Pyrazindiamin-Teilstruktur verlorengeht, wurden sie nicht weiter in Erwägung gezogen.

\section{Experimenteller Teil}

Schmelzpunkt: Kofler-Heiztischmikroskop, nicht korrigiert.

Die ein- und zweidimensionalen NMR-Spektren wurden mit einem Varian $400 \mathrm{MHz}$ Unity Inova Spektrometer mit inversem $5 \mathrm{~mm}$ Breitbandprobenkopf und Gradienteneinheit bei einer Meßfrequenz von $399.98 \mathrm{MHz}$ für ${ }^{1} \mathrm{H}$ und $100.59 \mathrm{MHz}$ für ${ }^{13} \mathrm{C}$ aufgenommen. Für die Durchführung der $g s-\mathrm{HMBC}^{12}$ - und 1D-NOE-Differenz ${ }^{13}$ Experimente wurden die Standard-Pulsprogramme verwendet. Die einzelnen $g s$-HMBC-Experimente wurde jeweils mit einem Delay von $125,83,63,50,42,31$ und $25 \mathrm{~ms}$ für ${ }^{n} J_{\mathrm{CH}}=4,6,8,10,12,16$ und $20 \mathrm{~Hz}$ optimiert. Für die NOE-Messungen wurden die Proben entgast und $T_{1}$-Bestimmungen durchgeführt. 5-10 mg Substanz wurden in ca. $0.5 \mathrm{ml}$ deuteriertem Lösungsmittel gelöst. Die Werte für die chemische Verschiebung sind in $\delta / \mathrm{ppm}$ mit TMS als internem Standard angegeben. I: Intensität. MS (EI) $(70 \mathrm{eV}$ ): Finnigan-MAT 8430 (Institut für Organische Chemie der TU Braunschweig); R.I.: relative Intensität. FT-IR: ATI Mattson Genesis Series UV-Vis: Philipps PU 8730 UV/VIS-Spektrophotometer. Fluorimeter: Kontron SFM 25. Reagenzien: Ph. Eur. 1997'. DC: Polygram ${ }^{1}$ SIL G/UV $254-$ Fertigfolien (Macherey-Nagel) $40 \times 80 \mathrm{~mm}$, Fliessmittel: Chloroform/Methanol =18:2. HPLC: L-6200A Gradientenpumpe, 
AS-2000A Autosampler, D-6000A Interface, L-7450 Dioden-array-Detektor, D-7000 HPLC System Manager (alle Geräte Fa. Merck und Hitachi), Säule LiChroCart 125-4, LiChrospher 100 RP-18 $(5 \mu \mathrm{m})$, Vorsäule LiChroCart 4-4, Select B, Flussrate $1.000 \mathrm{ml} / \mathrm{min}$.; isokratisch, Auftragevolumen $20 \mu \mathrm{l}$, Detektion $250 \mathrm{~nm}$, Nettoretentionszeit $\left(t_{\mathrm{s}}\right)$, Totzeit $\left(\mathrm{t}_{\mathrm{m}}\right)$ bestimmt mit Thioharnstoff, Eluent $\mathrm{H}_{2} \mathrm{O} / \mathrm{MeCN}=80 / 20$. CHN: C-H-N-O-Elemental Analyzer 1106 Carlo Erba. Röntgenstrukturanalyse: vollständige Daten (außer Strukturfaktoren) wurden unter der Nummer CCDC 168635 beim Cambridge Crystallographic Data Centre deponiert und können kostenlos angefordert werden vom Direktor, CCDC, 12 Union Rd., Cambridge. CB2 1EZ, UK. (e-mail: deposit@chemcrys.cam.ac.uk).

\section{3-(3-Amino-1,2,4-oxadiazol-5-yl)-5-chlor-2,6-pyrazindiamin (2)}

$0.2 \mathrm{~g}(0.66 \mathrm{mmol}) 1 . \mathrm{HCl}$ werden in $200 \mathrm{ml}$ Wasser gelöst. Zu der Lösung werden $15.5 \mathrm{ml}$ Natriumhydroxid-Lösung 8,5\% und $11 \mathrm{ml}$ Bromwasser R gegeben. Dann wird mit Salzsäure $36 \%$ auf $p \mathrm{H} 3.2$ eingestellt. Nach Zugabe von $25 \mathrm{ml}$ Natriumthiosulfat-Lösung $0.1 \mathrm{~mol} / \mathrm{l}$ wird mit $6 \times 100 \mathrm{ml}$ tert-Butylmethylether ausgeschüttelt. Die vereinigten organischen Phasen werden über Natriumsulfat getrocknet, i. Vak. abdestilliert und der Rückstand mit wenig Chloroform gewaschen und abgesaugt. Nach Umkristallisation aus $\mathrm{DMF} / \mathrm{H}_{2} \mathrm{O}$ erhält man $2 \mathrm{mg}$ (1\%) 3. Fp: ab $295^{\circ} \mathrm{C}$ (Zers.). IR (KBr): $\widetilde{v}=3486 \mathrm{~cm}^{-1}, 3471,3378,3333,3164$ $(\mathrm{N}-\mathrm{H}), 1662,1625,1604,1584(\mathrm{C}=\mathrm{N}, \mathrm{C}=\mathrm{C}) . \mathrm{UV}$ (Dioxan): $\lambda_{\max }(\lg \varepsilon)=231 \mathrm{~nm}(3.77)$, 282 (4.15), 368 (4.27). Fluoreszenz (Anregungswellenlänge $368 \mathrm{~nm}$ ): $\lambda_{\max }=417 \mathrm{~nm}$. DC: $\mathrm{Rf}=0.31$, blaue Fluoreszenz bei $365 \mathrm{~nm}$. HPLC: $\mathrm{t}_{\mathbf{s}}=1.47 \mathrm{~min}$. CHN für $\mathrm{C}_{6} \mathrm{H}_{6} \mathrm{ClN}_{7} \mathrm{O}$ (227.6). Ber: C 31.66 H 2.66 N 43.08; Gef: C 31.60 H 2.69 N 42.41.

\section{Literatur}

[1] Europäisches Arzneibuch 1997 (3. Ausgabe): 467, Deutscher Apotheker Verlag Stuttgart, Govi-Verlag - Pharmazeutischer Verlag GmbH Eschborn

[2] Shepard K.L., Halczenko W., Cragoe, E.J.,Jr. (1969) Tetrahedron Lett. 54: 4757

[3] Mazzo D.J. (1986) in Florey K. (Hrsg.) Analytical Profiles of Drug Substances, Bd. 15: 1, Academic. Press, New York

[4] Smith R.L., Cochran D.W., Gund P., Cragoe, E.J.,Jr. (1979) J. Am. Chem. Soc. 101: 191

[5] Hartke K., Hartke H., Mutschler E., Rücker G., Wichtl M. (1993) Kommentar zum Europäischen Arzneibuch: $A$ 31, Wissenschaftliche Verlagsgesellschaft $\mathrm{mbH}$ Stuttgart - Pharmazeutischer Verlag GmbH Eschborn 
[6] Sato N. (1989) J. Heterocycl. Chem. 26: 817

[7] Watthey J.W.H., Desai M., Rutledge R., Dotson R. (1980) J. Med. Chem. 23: 690

[8] Lessel J. (1993) Pharmazie 48: 812

[9] Shutske G.M., Agnew M.N. (1981) J. Heterocycl. Chem. 18: 1025

[10] Bock M.G., Smith R.L., Blaine E.H., Cragoe, E.J,Jr.(1986) J. Med. Chem. 29: 1540

[11] Gonczi C., Korbonits D., Harsanyi K. (1975) Chem. Abstr. 83: P131610k (Ger. Offen $2,461,882$ )

[12] Kessler H., Oschkinat H., Griesinger C., Bermel W. (1986) J. Magn. Reson. 70: 106

[13] Kinns M., Sanders J.K.M. (1984) J. Magn. Reson. 56: 518 